\title{
Aptitude, task performance, and solution strategy of the three-term series problem
}

\author{
HIROSHI YAMA ${ }^{1}$ \\ Department of Educational Psychology, Faculty of Education, Kyoto University, Sakyo-ku, Kyoto $606^{2}$ \\ TAKAO UMEMOTO and YOKO KINJŌ \\ Department of Psychologv, Facully of Literature, Konan Women's University, Higashinada-ku, Kobe 658
}

\begin{abstract}
The purpose of this study was to investigate relationship between aptitude, task performance, and solution strategy of the three-term series problem, which is one of the most simple deductive reasoning. A sample of 25 subjects was selected from a larger sample of 121 female college students. They had previously taken the Kyoto University SX Intelligence Test and participated in the experiment in which, two premises of the problem being presented successively, they indicated whether three terms were ordered linearly or not. Six alternative models each corresponding to different strategies were supposed and 16 of subjects were divided into four groups by testing which model each subject's data were fit to. The results were that the correlation between general intellectual aptitude and the performance was not significant, that some strategies were advantageous for accuracy, that subjects of some strategy groups had higher intellectual abilities, and that the best strategy for accuracy did not necessarily require the highest abilities to be executed. Aptitude didn't have a direct effect on performance, but on strategy.
\end{abstract}

Key words: strategy, aptitude, performance, the three-term series problem, deductive reasoning,

It is often observed that individuals solve cognitive tasks in different ways. It is also the case with the three-term series problem, which is one of the most simple indirect deductive reasoning tasks such as " $A$ is larger than $B$, and $B$ is larger than C. Which is the largest?" (Yama, 1987).

Cognitive aptitude is a psychological construct about such individual differences, and current researches on it have used information processing models to describe different cognitive processes (cf. Snow, 1978).

Yama proposed six alternative componential models (cf. Sternberg, 1977) for describing the process of combining information from two premises in the solu-

1 We would like to thank Prof. Noboru Sakano of Kyoto University for his helpful suggestions.

2 Now at Osaka Prefectural College of Nursing, Tezukayama-higashi, Sumiyoshi, Osaka 558. tion strategy. The task Yama set to his subjects was, when presented two premises successively, to decide whether three terms were ordered linearly or not, as rapidly as possible.

Three of the six models are linguistic models which have "pivot search in the second premise (PS2)" parameter. The parameter represents the mental operation required if the pivot is not the subject of the second premise. The other three are spatial models which have "endanchor (EA)" parameter. The parameter corresponds to the bringing process of the third term to the spatial array which is already constructed from the first premise, which is easy when the third term is the subject of the second premise. The value of the parameters and the problem types are shown in Table 1.

To each of the above parameters, one of the three parameters of the pivot 
tion of the three-term series problem. Each model corresponds to its own solusearch in the first premise are appended. If the first premise is retained word for word, the pivot search will be casier when the pivot is grammatically the same as in the second premise. The value of "grammatical pivot search (PSGR)" parameter reflects this difficulty. If the first premise is encoded into a propositional form, the search will be easier when the pivot is the subject. The value of "propositional pivot search (PSPR)" reflects this difficulty. If the terms of the first premise are seriated from the "larger" to the "smaller" in retaining, the search will be easier when the pivot is supposed to be the larger one in the first premise. The value of "directional pivot search (PSDI) " reflects this difficulty. Hence six models are proposed: PS2-GR, PS2PR, PS2-DI, EA-GR, EA-PR, and EADI models. Moreover, to each model, "lexical marking of the first premise (LM1)" and "lexical marking of the second premise (LM2)" parameters are appended to predict RT data of each subject more precisely. These parameters reflects the difficulty in processing "smaller".

The purpose of this study is to investigate relationships between the cognitive aptitude, task performance, and strategy choice of the three-term series problem. In this study, each specific ability score was created by its corresponding subtest of the Kyoto University SX Intelligence Test (SX) and general cognitive ability score was created by summing scores of all the subtests. These scores are the index of the aptitude. Reaction time and error rate was used as the index of the task performance (cf. Umemoto, 1987).

Kyllonen, Lohman, and Woltz (1984) classified aptitude-strategy relations into three types. The first is that strategy selection is limited by aptitude (Egan and Grimes-Farrow, 1982). The second is that it is not strategy selection but per- formance that is related to aptitude (Sternberg and Weil, 1980). The third is the combined case of the first and the second: aptitude limits both strategy and task performance (MacLeod, Hunt, and Mathews, 1978), which was supported by Kyllonen et al. (1984).

In the case of this study, the strategies differ in constructed representation (linguistic or spatial), and in memory demand. For example, the processing PSGR parameter represents needs greater memory capacity than that PSDI parameter does, because PSGR parameter corresponds to the mental operation in which the first premise is retained word for word, while PSDI parameter corresponds to the operation in which the comparative of the first premise is not processed. Hence, it is predicted that the strategy selection will be limited by the aptitude, and that the aptitude will affect the performance, because speed and accuracy are demanded in both the task and SX, which is the third type prediction of Kyllonen et al. (1984).

\section{Method}

Subjects. A random sample of $25 \mathrm{fe}$ male students was selected from a larger sample of 121 Konan Women's College undergraduates. Subjects had previously taken the SX including following subtests: sentence completion, sentence reasoning, sentence re-arrangement, word classification, word completion, homonym production in Chinese character, code exchanging, calculation, figure reconstruction, dot figure, matrix matching, and punched hole. This test is for those who are high in cognitive aptitude, particularly for college students, hence it is a little more difficult than those usually used in school.

Stimuli. The following stimuli were used. The letters " $F$ ", " $N$ ", and " $S$ " were used as three terms and "largersmaller" as comparatives. The eight 
Table 1

Problem types, value of variables multiplying parameters, mean reaction times for correct responses, and error rates

\begin{tabular}{|c|c|c|c|c|c|c|c|c|c|}
\hline \multirow{2}{*}{ Premises } & \multicolumn{7}{|c|}{ Parameters } & \multirow{2}{*}{$\begin{array}{c}\text { Mean } \\
\text { RTs }\end{array}$} & \multirow{2}{*}{$\begin{array}{l}\text { Error } \\
\text { rates }\end{array}$} \\
\hline & PS2 & EA & PSGR & PSPR & PSDI & LMI & LM2 & & \\
\hline $\mathrm{A}>\mathrm{B} \quad \mathrm{B}>\mathrm{C}$ & 0 & 1 & 1 & 1 & 1 & 0 & 0 & 3358 & .43 \\
\hline $\mathrm{A}>\mathrm{B}, \mathrm{C}<\mathrm{B}$ & 1 & 0 & 0 & 1 & 1 & 0 & 1 & 3360 & .26 \\
\hline$B<A, B>C$ & 0 & 1 & 0 & 0 & 1 & 1 & 0 & 3257 & .33 \\
\hline $\mathrm{B}<\mathrm{A}, \mathrm{C}<\mathrm{B}$ & 1 & 0 & 1 & 0 & 1 & 1 & 1 & 3293 & .35 \\
\hline $\mathrm{B}>\mathrm{C}, \mathrm{A}>\mathrm{B}$ & 1 & 0 & 1 & 0 & 0 & 0 & 0 & 3198 & .37 \\
\hline $\mathrm{B}>\mathrm{C}, \mathrm{B}<\mathrm{A}$ & 0 & 1 & 0 & 0 & 0 & 0 & 1 & 3342 & .34 \\
\hline $\mathrm{C}<\mathrm{B}, \mathrm{A}>\mathrm{B}$ & 1 & 0 & 0 & 1 & 0 & 1 & 0 & 3024 & .28 \\
\hline$C<B, B<A$ & 0 & 1 & 1 & 1 & 0 & 1 & I & 3426 & .46 \\
\hline
\end{tabular}

Note. PS2 = pivot search in the second premise; EA=end-anchor; PSGR = grammatical pivot search ; PSPR = propositional pivot search; PSDI=directional pivot search ; LMI=lexical marking of the first premise; $L M 2=$ lexical marking of the second premise.

All reaction times are expressed in milliseconds.

problem types, shown in Table 1 , were constructed by varying whether (1) the adjective in the first premise was "larger" or "smaller", (2) the adjective in the second premise was "larger" or " smaller", (3) the order of two premises was "A-B, B-C", or "B-C, A-B". Eight partial order problem types were constructed through exchanging the adjectives of the second premises of the linear order problems. Moreover, six kinds of problems were made by changing the order of the three letters for each type. Hence the number of the problems was 96.

Procedure. Subjects participated in the experiment individually. All the premises were presented in NEC-N5913 color display, controlled by NEC-9801VX. Each trial started after subjects pushed the middle key to indicate that they were ready. After a second's buzzer, the first premise was presented for three seconds. When it was turned off, the second premise was presented. From that time, subjects had to decide whether the three terms were ordered linearly or not, and respond by pushing one of two buttons, right or left. RTs were measured in milliseconds. If they couldn't respond in six seconds, the second premise was turned off, and the response was regarded as error even if they responded correctly. ${ }^{3}$

\section{Results}

Mean RTs of correct responses and error rates for each problem are shown in Table 1.

\section{Testing of Models}

As dependent variable of each subject, median of correct RTs for each problem type was used. The proportion of variance in each subject's data accounted for by each model $\left(R^{2}\right)$ was calculated by stepwise multiple regression. The subjects were grouped in the following ways. First, subjects whose data were fit to PS2 parameter were assigned to linguistic groups, while those whose data were fit to EA parameter were assigned to spatial groups. Next, for each subject, one of the pivot search in the first premise parameters whose estimate was positive, and whose $R^{2}$ was the highest was regarded

${ }^{3}$ This criterion might be a little harsh to the subjects, but when RT was longer than the criterion, they often reported that they had forgotten the first premise. 
Table 2

Number of subjects of each group and $R^{2}$ in RTs data attributable to parameters for each group

\begin{tabular}{lcccccc}
\hline $\begin{array}{l}\text { Model } \\
\text { Group }\end{array}$ & $\begin{array}{c}\text { Number of } \\
\text { subjects }\end{array}$ & PS2/EA & PS (GR PR DI) & LM1 & LM2 & $\begin{array}{c}\text { Total } \\
R^{2}\end{array}$ \\
\hline PS2-GR & 6 & .18 & $.62^{*}$ & .01 & - & $.81^{*}$ \\
PS2-DI & 2 & .08 & .45 & - & .14 & .67 \\
PS2 & 2 & .01 & - & - & $.51^{*}$ & .52 \\
EA-GR & 4 & .35 & .45 & .10 & .07 & $.97^{* *}$ \\
EA-PR & 1 & .00 & .30 & .00 & .13 & .51 \\
EA-DI & 4 & .02 & $.66_{*}$ & - & - & $.68^{*}$ \\
EA & 6 & .40 & - & - & .12 & .52
\end{tabular}

Note. Model groups of PS2 and EA do not have an optimal parameter of the pivot search in the first premise.

as her optimal parameter, only that the $R^{2}$ of that was less than .1. In this way each subject's optimal model was determined, though eight subjects did not have optimal parameter of pivot search in the first premise. All the subjects were divided into seven groups for their optimal models. No subjects were assigned to PS2-PR model. The number of subjects and $R^{2}$ of each optimal model for the mean RT data of each group are shown in Table 2. Because all the parameters are independent in each model, its $R^{2}$ is the sum of each parameter's $R^{2}$.

\section{Relationship}

First, we examined the relationship between the aptitude and the performance. The sum of the subscores of SX, which represents a subject's general cognitive aptitude, was not significantly correlated with inner-subject mean RT $[r(24)=$ $-.04]$, nor with error rate $[r(24)=.01]$. These results suggests that the aptitude had an effect on neither speed nor accuracy of this task.

Second, we examined the relationship between the aptitude and the strategy choice. Because the number of subjects in EA-PR model was only one, we compared those of PS2-GR, PS2-DI, EA-GR, and EA-DI models. We computed 4 (groups) $\times 12$ (subtests) analysis of vari-
Table 3

Mean reaction time, error rate, and $Z$-scores of Intelligence Test for each group

\begin{tabular}{lccc}
\hline Groups & RTs & Error rates & $Z$-scores \\
\hline PS2-GR & 3142 & .41 & -.03 \\
PS2-DI & 3160 & .14 & -.53 \\
PS2 & 3302 & .29 & -1.53 \\
EA-GR & 3215 & .33 & 1.05 \\
EA-PR & 3907 & .46 & .08 \\
EA-DI & 3309 & .29 & -.20 \\
EA & 3378 & .41 & .02 \\
\multicolumn{1}{c}{ Total } & 3282 & .35 & .00
\end{tabular}

ance. The main effect of grouping was significant $[F(3,12)=16.72, p<.001]$, but, through Ryan's $t$-test of multiple comparison, no difference between any two groups was significant. The $Z$-scores of the sum of the subtests for all strategy groups is shown in Table 3. Generally, subjects of the EA groups had higher aptitude than those of the PS2 groups, and subjects of the PSGR groups had higher aptitude than those of the PSDI groups. It was suggested that some strategies required higher aptitude to be executed than others.

The grouping by these parameters did not significantly interacted with the subtest variable, not supporting Yama's supposition that PS2 parameter was related to linguistic process, while EA parameter 
was related to spatial process.

Finally, the relationship between performance and strategy was examined. For comparing the performance of subjects in the different strategy groups, the means of $\mathrm{RT}$ and error rates are shown in Table 3. Generally, the mean RTs of the subjects of PSGR groups were a little smaller than those of other groups, though the differences were not significant. But the error rates differed each other remarkably. The effect of grouping was significant $[F(3,12)=79.33, p<$ $.001]$. Generally the subjects in the PSDI groups, particularly in the PS2-DI group, made less errors, though, through Ryan's $t$-test of multiple comparison, no differences were significant. All considered, we assert that some strategies were more effective than others in accuracy.

\section{Discussion}

The major findings of this study were that different subjects used different strategies for solving the three-term series problem, that though the general cognitive aptitude was not related to the performance, some strategies required higher cognitive aptitude, and some strategies were better than others in that they led to more accurate performance, but that the best strategy for accuracy was not the one which required the highest aptitude.

First, we will discuss why some strategies brought better performance in accuracy. One factor, perhaps the most important one, is the working memory capacity (Johnson-Laird, 1988) each strategy needs. Because the processing PSGR parameter represents needs greater capacity than that PSDI parameter does, the subjects in PSDI groups made less errors. But this factor was not effective in the EA model groups, and in these groups, the error rates were medium. We can not explain the reason clearly, but perhaps, because the processing corresponding to EA parameter is visuo-spatial, hence the capacity limit does not affect the processing corresponding to PSGR parameter.

The main interest was in the relationship between strategy and aptitude. According to the hypothesis of our models, subjects whose spatial aptitude is comparatively higher than his or her linguistic aptitude would choose spatial strategies. But it was not supported statistically. One tendency was that, generally, the subjects of the EA groups had higher aptitude than those of PS2 groups. Perhaps this was partially because of the nature of the aptitude measured by SX. SX is the intelligence test for high aptitudes, so it is more difficult than any other test. The subtest tasks for linguistic aptitude might be solved with the aid of spatial aptitude. This tendency was also observed in the results of MacLeod, Hunt, and Mathews (1978). Second, the subjects of PSGR groups had the higher general cognitive aptitude than those of PSDI groups. As noted above, this processing needs greater memory capacity. But at the same time it does not need mental effort to encode the first premise into more simple form. Hence, the subjects who had the high aptitude chose the PSGR strategies.

These explanations will make readers reason why the strategy corresponding to EA-GR model, which required the highest aptitude was not the best one for accuracy. The PSGR strategies, though not significant, are nevertheless advantageous to speed, but disadvantageous to accuracy. The strategy which requires the highest aptitude is not necessarily the best one for performance.

In short, aptitude was not directly related to performance, but constrained strategy choice, and individual differences in accuracy were mostly due to strategic difference. These results of this study propose a new type of relations that aptitude limits strategy and that the strategy limits task performance. 


\section{References}

Egan, D. E. \& Grimes-Farrow, D. D. 1982 Differences in mental representations spontaneously adopted for reasoning. Memory and Cognition, 10, 297-307.

Kyllonen, P. C., Lohman, D. F., \& Woltz, D. J 1984 Componential modeling of alternative strategies for performing spatial tasks. Journal of Educational Psychology, 76, 1325-1345.

Johnson-Laird, P. N. 1988 The computer and the mind. Cambridge, Mass: Harvard University Press.

Macheod, C. M., Hunt, E. B., \& Mathews, N. N. 1978 Individual differences in the verification of sentence-picture relationships. Journal of Verbal Learning and Verbal Behavior, 17, 493-507.

Snow, R. E. 1978 Theory and method for re- search on aptitude processes. Intelligence, 2, 225-278.

Sternberg, R. J. 1977 Intelligence, information processing, and analogical reasoning: The componential analysis of human abilities. Hillsdale, NJ: Erlbaum.

Sternberg, R. J., \& Weil, E. M. 1980 An aptitude-strategy interaction in linear syllogistic rcasoning. Journal of Educational Psychology, 109, $119-159$.

Umemoto, T. 1987 Cognilion and performance. Vol. 6. Cognitive science series. University of Tokyo Press. (In Japanese)

Yama, H. 1987 Linguistic and spatial processings in solving three-term series problem. Japanese Psychological Research, 29, 197-201.

(Received July 8, 1988; accepted Sept. 9, 1989) 